\title{
Comparison of Ortho-planar Spring design optimization based on Linear Elastic and Hyper Elastic Materials
}

\author{
Ruetai Graipaspong ${ }^{1}$ and Teeranoot Chanthasopeephan ${ }^{1}$ \\ ${ }^{1}$ Department of Mechanical Engineering, King Mongkut's University of Technology Thonburi, Bangkok, 10140 Thailand
}

\begin{abstract}
In this paper, compliant Ortho-planar spring was designed based on a three-dimensional topology optimization method. The computation was developed using MATLAB programming. The objective of this work was to apply dual method to design an Ortho-planar spring while the design should have minimum mass and at the same time satisfy a set of constrained displacement. Throughout this paper, we analyzed a method for designing an Ortho-planar spring using linear elastic material and hyperelastic material. The results showed that under small displacement conditions, the output displacement, maximum stress magnitude, and the maximum stress of linear elastic assumption and hyper-elastic material were relatively close to each other. However, the mass fraction and the layout as the result of the optimization process was different. As for larger displacement, the maximum stress of linear elastic material appeared 2.59 times higher than the maximum stress of the hyper-elastic material model. The topology optimization output based on linear material was invalid because the topology of the computed Ortho-planar spring was not appeared as a one-piece layout while the design based on nonlinear material looked promising.
\end{abstract}

\section{Introduction}

Ortho-planar spring in general is described as a mechanism that lifts the platform up from its plane. Compliant mechanism, on the other hand, is a mechanism moved by deflection of members. Instead of using joint to lift the platform, compliant Ortho-planar spring moves through the deflection of members $[1,2]$. The avantages of compliant Ortho-planar springs are not only the ease of manufacturing process, but also that their compactness, resistance to wear, and light weight [2]. Multiple applications of Ortho-planar springs include valves, actuators, pneumatic controllers, etc. [3-5]. In each application, the layouts of the compliant Ortho-planar springs are designed differently.

Topology optimization is a mathematical approach that synthesizes the optimum layout of the structures within the given loads and boundary conditions. In the past, various calculation tools were used for topology optimization. Mathematica had been used to design Michell truss structures [6]. Aside from Mathematica, MATLAB was also used for calculation. Through MATLAB implementation, Sigmund [7] introduced 99line code for 2-D topology optimization using optimum criteria method. Afterward, the 99-line code was shortened by Andreassen [8] until the code reached 88 lines. One advantage of decreasing lines of the codes was it took less time to synthesize the optimal layout. Although the 88-line MATLAB code successfully reduced computational time, the computation was limited to only two-dimensional design. As a result, Liu [9] had developed a three-dimensional topology optimization MATLAB code using optimum criteria method. The designs of Sigmund, Andreassen, and Liu were based on optimum criteria method, where the objective function was to minimize compliance while limited the volume fraction. The limitation of volume fraction was used as a condition while computing for the structure with the desired weight. Aside from using minimize compliance as the objective function, other objective functions could still be used. Sui [10] applied dual method for 2dimensional topology optimization with the objective function of mass minimization instead of compliance minimization. Sui's constrained the displacement of the loaded structure, not the volume fraction, unlike the above mentioned works. By using different calculation method, different objective functions could be appropriately chosen for each case.

Although many topology optimization models worked well with linear finite element, it could not be used for large displacement analysis where nonlinearity plays an important role. Nonlinearity analysis usually suits with geometrically nonlinear, material nonlinear, or combined nonlinear problems. The analysis and design of nonlinear compliant mechanisms using hyperelastic materials was explored by Bruns [11]. Nonlinear hyperelastic material was required for large displacement compliant mechanisms. The formulated equations were solved by using Newton-Raphson method. Jung [12] discussed the differences between the optimal layouts and deformed shapes of linear design, materially nonlinear design, geometrically nonlinear design, and combined design. 
Nonlinear material was not only modelled by using functions between stress and strain, but also from deriving constitutive equations in terms of second Piola Kirchoff stress and Green-Lagrange strain tensors. Topology optimization of compliant mechanisms based on nonlinear design using meshless models was done by $\mathrm{Du}$ [13]. Their method suited well for large deformation of a compliant mechanism design. Meshless models discretized the design domain by nodes, instead of elements. The proposed numerical optimization process could be used for finite element method as well.

In this paper, compliant Ortho-planar spring was designed based by developing a MATLAB code to compute for three-dimensional topology optimization. Dual method was applied and the objective function was to minimize mass. The constraint was a define set of displacement. The aim of this research was to analyze and compare the design of an Ortho-planar spring based on linear elastic material versus hyper-elastic material.

\section{Topology optimization}

The main steps of topology optimization are presented in the flowchart as shown in Figure 1. According to the flow chart, the steps toward topology optimization process in Figure 1 were given as follow. To design an Ortho-planar spring, the first step started with setting a specified set of length, width, and height of the prototype. Then, an objective function which subjected to its constraint was constructed. The initial conditions were set. To prevent checkerboard structure, meshindependency filter was added to the process.

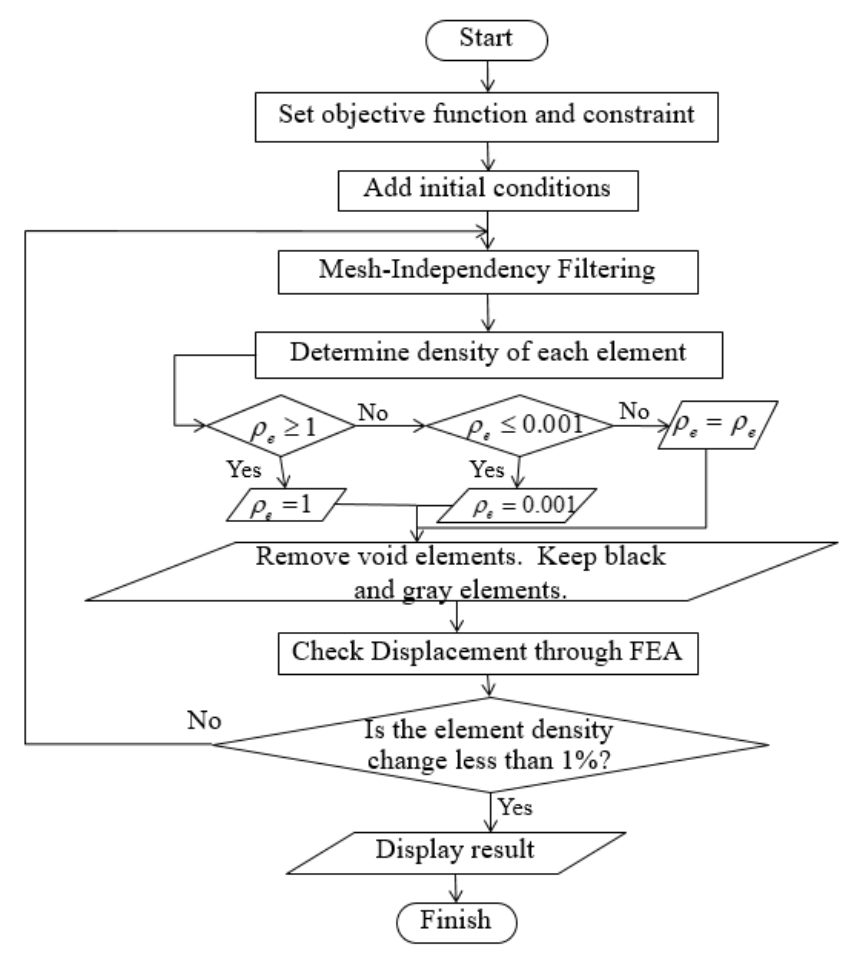

Figure 1. Algorithm for topology optimization

The following steps start with determine element density, remove elements that have element density of $\rho_{\min }$ (void elements), and keep the elements with element density in the range between $\rho_{\min }$ and 1 (gray and black elements). As the element density was calculated, the outcome of the displacement should be checked whether it has the same value as the constraint or not. Afterward, the element density of the current iteration is compared to the element density in previous iteration. If either changes in element density is higher than 1 percent or the output displacement contradicts the constraint, the process has to be repeated. Once the output displacement and change in element density satisfies the conditions, the calculation is finished.

In this paper, the objective function is to minimize mass, while constraining the displacement of Orthoplanar spring. The summation of element mass, $m_{e}$, lead to total mass, $m$, which depends on element density, $\rho_{e}$. The element density must be between minimum density and 1 .

$$
\begin{array}{ll}
\min & m(\rho)=\sum_{e=1}^{N} \rho_{e}(\rho v)_{e}=\sum_{e=1}^{N} \rho_{e} m_{e} \\
\text { s.t. } & u(\rho)=\bar{u} \\
& 0<\rho_{\min } \leq \rho_{e} \leq 1
\end{array}
$$

According to the work of Sui [10], the structure displacement $u(\rho)$ is the sum of element displacement. From work-energy theorem and stiffness matrix displacement is obtained as

$$
u=\sum_{e=1}^{N}\left(P_{e}^{R}\right)^{T}\left(K_{e}^{0}\right)^{-1} P_{e}^{v} \rho_{e}^{-p} \approx \sum_{e=1}^{N} \frac{D_{e}^{0}}{\rho_{e}^{p}}
$$

where $p$ and $D_{e}^{0}$ are the density penalty and displacement coefficient respectively. $K_{e}^{0}$ and $P_{e}^{v}$ are the element stiffness matrix per unit element density and element nodal force associated to the virtual load respectively. The value of input penalty depends on the Poisson's ratio of the selected material. If the input penalty is not high enough, the solution may not reach its global optimum[14]. Therefore, the objective function is as following:

$$
\begin{array}{ll}
\min & m(\rho)=\sum_{e=1}^{N} \rho_{e}(\rho v)_{e}=\sum_{e=1}^{N} \rho_{e} m_{e} \\
\text { s.t. } & \sum_{e=1}^{N} \frac{D_{e}^{0}}{\rho_{e}^{p}}=\bar{u} \\
& 0<\rho_{\min } \leq \rho_{e} \leq 1
\end{array}
$$

Using a Lagrange multiplier to combine the equations and solving the Lagrangian equations, element density equation is then obtained. 


$$
\rho_{e}=\left(\frac{\sum_{k=1}^{N}\left(D_{k}^{0}\right)^{\frac{1}{p+1}}\left(m_{k}^{0}\right)^{\frac{1}{p+1}}}{\bar{u}}\right)^{\frac{1}{p}} \cdot\left(\frac{D_{e}^{0}}{m_{e}^{0}}\right)^{\frac{1}{p+1}}
$$

The element density limit has been given between $\rho_{\text {min }}$ and 1 . Therefore, the element density is equal to 1 , or element is solid, when the calculated density value exceeds 1 . Moreover, it is given at the minimum value or as void, if the density from calculated from the equation is less than or equal to $\rho_{\min }$. The element is present but would have gray color as the calculated value is between $\rho_{\min }$ and 1 .

\section{Linear material and nonlinear hyperelastic material analysis}

\subsection{Linear Material Analysis}

The difference between linear material and nonlinear material analysis is the stiffness matrix. With linear material assumption, the stiffness matrix was considered as a constant matrix. The element stiffness matrix was in the form of

$$
K e_{\text {linear }}=\int_{V} B^{T} C(E, v) B d V
$$

where $B$ and $C$ were strain-displacement matrix and constitutive matrix respectively. In linear finite element analysis, the constitutive matrix was a function of Young's modulus $(E)$ and Poisson's ratio $(v)$.

\subsection{Nonlinear Hyperelastic Material Analysis}

Equation(5) could not be used to determine the element stiffness matrix. In the case of nonlinear material with linear geometry assumption, the stiffness matrix depended on displacement. The tangent element stiffness matrix was described in the form of

$$
K e_{\text {nonlinear }}=\int_{V} B_{m}{ }^{T} S B_{m} d V
$$

where $B_{m}$ was the strain-displacement matrix for nonlinear material. The $S$ matrix was the second Piola Kirchoff stress matrix, in which the values depend on the displacement of the structure. Second Piola Kirchoff stress was calculated from the models that were used to fit the stress-strain curve. There were multiple models that could be used to fit the hyper-elastic material stressstrain curve, depending on each case. In this paper, Yeoh's model was applied.

$$
W=c_{1}\left(I_{1}-3\right)+c_{2}\left(I_{1}-3\right)^{2}+c_{3}\left(I_{1}-3\right)^{3}
$$

where $W$ and $I_{1}$ are strain energy and first invariant respectively. Aside from that, $c_{1}, c_{2}$, and $c_{3}$ were constants. From strain energy equation, second Piola Kirchoff stress was obtained by

$$
S=c_{1} I_{1, E}+2 c_{2}\left(I_{1}-3\right) I_{1, E}+3 c_{3}\left(I_{1}-3\right)^{2} I_{1, E}
$$

where $I_{1, E}$ was the derivative of first invariant.

As it was previously mentioned, the stiffness matrix of hyper-elastic materials depended on the displacement. Therefore, the finite element equation could not be solved directly. Various numerical methods have been mentioned to solve nonlinear finite element models. The most suitable method for this case was Newton-Raphson method [15, 16]. This strategy worked by using the tangent stiffness matrix " $K e_{\text {nonlinear }}$ " and the residual force " $R$ " to find the displacement of the iteration.

$$
\begin{gathered}
R\left(U_{x-1}\right)=\left[K_{\text {nonlinear }}\left(U_{x-1}\right)\right]\left(\mathrm{U}_{x-1}\right)-F \\
R\left(U_{x-1}\right)=\left[K_{\text {nonlinear }}\left(U_{x-1}\right)\right](\Delta \mathrm{U})
\end{gathered}
$$

The displacement of the current iteration, $x$, was found to be $U_{x}=U_{x-1}+\Delta U$. Since residual force was the difference between internal and external force, the loop continued until the residual force reached zero.

\section{Results and discussion}

The topology design of Ortho-planar spring had been calculated as load " $F$ " was applied in the center of a $50 \mathrm{~mm} \times 50 \mathrm{~mm} \times 1 \mathrm{~mm}$ solid structure. Each element was a given size of $1 \mathrm{~mm} \times 1 \mathrm{~mm} \times 1 \mathrm{~mm}$. Thus, the number of element along the $\mathrm{x}, \mathrm{y}$, and $\mathrm{z}$ directions were 50,1, and 50 respectively. Figure 2 showed that all four sides (blank surfaces) of the initial design were fixed, while the shaded surfaces represented free surfaces.

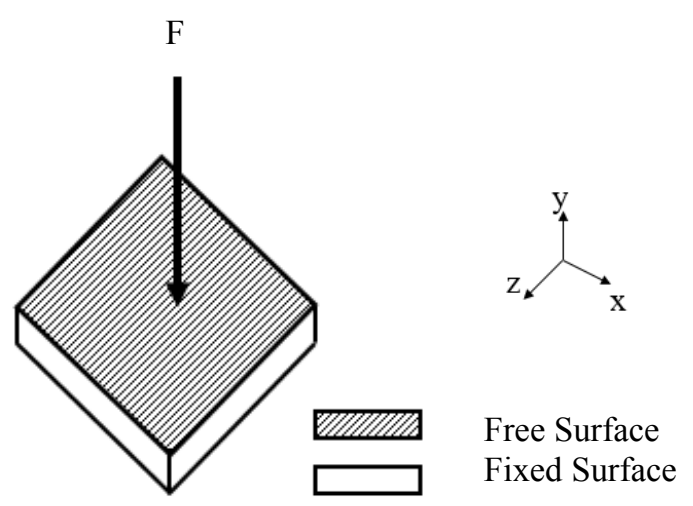

Figure 2. Initial condition of Ortho-planar spring 
Table 1. Results from small displacement input

\begin{tabular}{|l|c|c|}
\hline & Linear Elastic Material & Hyper-Elastic Material \\
\hline Output Displacement (mm) & 1.09 & 1.099 \\
\hline Maximum Stress (MPa) & 9.210 & 11.115 \\
\hline Mass Fraction & 0.3 & 0.25 \\
\hline & &
\end{tabular}

*The circles represent points of maximum stress.

Table 2. Results from large displacement input

\begin{tabular}{|l|c|c|}
\hline & Linear Elastic Material & Hyper-Elastic Material \\
\hline Output Displacement (mm) & 5.00 & 5.01 \\
\hline Maximum Stress (MPa) & 28.835 & 0.07 \\
\hline Mass Fraction & 0.1 & (2) \\
\hline & &
\end{tabular}

*The circles represent points of maximum stress.

Table 1 and Table 2 presented the results from small displacement and large displacement of the linear and nonlinear materials. In Table 1, the displacement was limited to $1.1 \mathrm{~mm}$. The maximum stress and the location of the maximum stress of linear elastic material and hyper-elastic material were close to each other. However, the outcome of mass fraction and layout were somewhat different.

In Table 2, the displacement was limited to $5 \mathrm{~mm}$. Although the locations of maximum stress of both cases were close to each other, the maximum stress of linear elastic material was 2.59 times higher than the maximum stress as the result of the hyper-elastic material model. This was due to the behavior of the material; hyperelastic materials have different behavior as compared to the linear elastic material as the displacement increases. Aside from that, the layout of the linear elastic material did not come out as one piece layout. The fragmented layout was invalid. At large strain, it was recommended to use nonlinear material properties to design Orthoplanar spring.

\section{Conclusion}

The applications of Ortho-planar spring include valves, actuators, pneumatic controllers, etc. In this paper, an Ortho-planar spring was designed through a topology optimization. With the given boundary conditions, the comparison between the effects of linear material property and nonlinear material property on topology optimization had been analyzed.

First, the displacement constraint was set as $1.1 \mathrm{~mm}$. The output displacement, maximum stress, and positions of maximum stress of linear elastic assumption and hyper-elastic material tend to be the same while the mass fraction and the layout were different. Then, the displacement constraint was set to $5 \mathrm{~mm}$. The output displacement, mass fraction, and positions of maximum stress tend to be the same. With larger displacement, the maximum stress of linear elastic assumption was 2.59 times higher than the maximum stress of the hyper-elastic material model. This was due to the different between the behavior of materials. Hyperelastic materials have 
different behavior than linear elastic material as the displacement increases. Also, the layout based on the linear elastic material assumption did not come out as a one piece layout, which meant that the layout of linear material case was invalid. Therefore, it was recommended to use nonlinear material properties to design Ortho-planar spring as large deformation occurred.

\section{Acknowledgement}

The authors would like to express gratitude to Smart DVision Company Limited and the Research and Researchers for Industries (RRI) scholarship No. MSD56I0018 under Thailand Research Fund (TRF) for financial support. We also thank Mechanical Engineering department, King Mongkut's University of Technology Thonburi for providing the equipment for testing.

\section{References}

1. J. J. Parise, L. L. Howell and S. P. Magleby, Mechanism and Machine Theory 36 (11-12), 12811299 (2001).

2. L. L. Howell, Compliant mechanisms. (John Wiley \& Sons, 2001).

3. B. D. Olivier Smal, Benoit Raucent, Michael De Volder, Jan Peirs, Dominick Reynaerts, Frederik Ceyssens, Johan Coosemans, Robert Puers, Journal of Micro-Nano Mechatronics 4 (3), 131-143 (2008).

4. O. Smal, B. Raucent, F. Ceyssens, R. Puers, M. Volder and D. Reynaerts, Micro-Assembly
Technologies and Applications: IFIP TC5 WG5.5 Fourth International Precision Assembly Seminar (IPAS'2008) Chamonix, France February 10-13, 2008, 75-86 (2008).

5. B. R. a. H. J. Olivier Smal, International Journal for Simulation and Disciplinary Design Optimization 3 (2), 356 - 362 (2009).

6. T. Sokól, Structural and Multidisciplinary Optimization 43 (2), 181-190 (2011).

7. O. Sigmund, Struct. Multidiscip. Optim. 21 (2), 120127 (2001).

8. E. Andreassen, A. Clausen, M. Schevenels, B. S. Lazarov and O. Sigmund, Structural and Multidisciplinary Optimization 43 (1), 1-16 (2011).

9. K. Liu and A. Tovar, Structural and Multidisciplinary Optimization 50 (6), 1175-1196 (2014).

10. G. Y. Yunkang Sui, in 10th World Congress on Structural and Multidisciplinary Optimization (2013).

11. T. E. Bruns and D. A. Tortorelli, Computer Methods in Applied Mechanics and Engineering 190 (26), 3443-3459 (2001).

12. D. Jung and H. C. Gea, Finite Elements in Analysis and Design 40 (11), 1417-1427 (2004).

13. L. C. Yixian Du, International Journal of CAD/CAM 8 (1), 1-10 (2008).

14. M. P. B. O. Sigmund, Topology optimization: theory, methods, and applications. (Springer, 2003).

15. J. N. Reddy, An Introduction to Nonlinear Finite Element Analysis. (OUP Oxford, 2004).

16. N.-H. Kim, Introduction to Nonlinear Finite Element Analysis. (Springer US, 2015). 